\title{
Adult-Onset Still's Disease: A Case Report
}

Review began 12/30/2021 Review ended 01/06/2022 Published 01/08/2022

\section{(๑) Copyright 2022}

Shad et al. This is an open access article distributed under the terms of the Creative Commons Attribution License CC-BY 4.0., which permits unrestricted use, distribution, and reproduction in any medium, provided the original author and source are credited.
Iram Shad $^{1}$, Muhammad Shafique ${ }^{1}$, Syeda A. Waris ${ }^{1}$, Farwa Shabbir ${ }^{1}$, Attiya Begum ${ }^{2}$

1. Internal Medicine, Holy Family Hospital, Rawalpindi, PAK 2. Obstetrics and Gynaecology, District Headquarters Hospital, Rawalpindi, PAK

Corresponding author: Syeda A. Waris, aimenwaris@hotmail.com

\section{Abstract}

Adult-onset Still's disease (AOSD) is a disorder that is occasionally seen. Autoinflammation is a generally accepted pathogenic mechanism leading to systemic signs and symptoms. We report the case of a young female presenting with high-grade fever, rash, and arthralgias. After a thorough assessment, the diagnosis of adult-onset Still's disease was made based on presenting symptoms and elevated serum ferritin, creactive protein (CRP), and absence of serologic markers and confirmed based on Yamaguchi criteria. She was treated with corticosteroids and achieved complete clinical remission.

Categories: Internal Medicine

Keywords: negative serological markers, steroids, yamaguchi criteria, autoinflammation, adult onset stills disease

\section{Introduction}

Adult-onset Still's disease (AOSD), with an incidence of one and 34 cases per million, is among one of the few unconventional causes of pyrexia of unknown origin [1]. The disease usually goes misdiagnosed, as its clinical features are indistinguishable from other causes of febrile illness. The most widely accepted pathogenic mechanism for this disorder is autoinflammation, in which there is an activation of the innate immune system against itself in response to danger signals [2]. There are no specific biomarkers that support the diagnosis. Since AOSD is a diagnosis of exclusion, labeling the patient with this disease requires a lot of work-up to rule out other differentials that appear alike, including infections, malignancy, and other rheumatic diseases [3]. We describe here a case of a young female patient of AOSD who has now achieved remission.

\section{Case Presentation}

A 12-year-old girl presented to the medical department of a tertiary care hospital with a complaint of fever for 14 days. Fever was sudden in onset, up to $104^{\circ} \mathrm{F}$, continuous, associated with rigors and chills, and partially relieved by antipyretics. Other symptoms included nausea, pain in the oligoarticular joints, maculopapular rash on the chest, and significant weight loss in the past few months. She had no history of prior hospitalization and was fully vaccinated according to the extended program immunization schedule. Physical examination was significant for the patient being lean and lethargic, with a fever and maculopapular salmon-colored rash on the chest with cervical lymphadenopathy.

Laboratory studies were significant for a markedly raised WBC count of $21.9 \times 10^{\wedge} 9$, platelets $506 \times 10^{\wedge} 3$, and mild anemia with hemoglobin of $8.8 \mathrm{~g} / \mathrm{dl}$. Tests of liver and renal function, prothrombin time (PT), and activated partial thromboplastin time (APTT) were normal. Keeping infective etiologies like urinary tract infection, infective endocarditis, tuberculosis, and brucellosis on top of the list of differential diagnoses, we went for a routine urine examination, urine and blood cultures, echocardiography, brucella antigens, and a chest X-ray, all of which came normal.

We then started workup to rule out malignant causes for which blood peripheral film with retics was sent, which showed neutrophilic leukocytosis and normal retics. Ultrasound examination of the abdomen and neck showed borderline splenomegaly and multiple enlarged cervical lymph nodes, more on the left side, with the largest measuring $13 \times 5.7 \mathrm{~mm}$ in size. Fine-needle aspiration cytology was done, which showed reactive hyperplasia. Next up, in order to rule out autoimmune causes, rheumatoid factor (RA), antinuclear antibody (ANA) levels, and erythrocyte sedimentation rate (ESR) were sent, all of which came normal with results of (<8IU/mL), $(<0.50)$, and $(22 \mathrm{~mm} / 1 \mathrm{hr})$, respectively. A list of common differentials that were ruled out is shown in Table 1 . 


\section{Cureus}

\section{Infections}

Urinary tract infection

Infective endocarditis

Tuberculosis

Brucellosis

Malignancies

Acute lymphoblastic leukemia

Hodgkins/Nonhodgkins lymphoma

Autoimmune

SLE, dermatomyositis, polymyositis

Post-streptococcal arthritis
Normal urine routine examination

Normal echocardiography, blood cultures showed no growth

Normal chest X-ray, no granulomatous changes in FNAC

Negative brucella antibodies

Normal peripheral film and retics count

No particular findings in FNAC

Negative ANA

Negative RA factor

\section{TABLE 1: Differential diagnoses that were ruled out}

FNAC: fine-needle aspiration cytology; ANA: antinuclear antibodies; RA: rheumatoid factor; SLE: systemic lupus erythematosus

With the common causes being ruled out, we started to think of AOSD. Serum ferritin and c-reactive protein (CRP) were sent as supportive evidence. Interestingly, both came high (5506 ng/ml and $98.4 \mathrm{mg} / \mathrm{L})$, respectively. Thus, our history, clinical findings, and investigations fulfilled Yamaguchi criteria for adultonset Still's disease.

\section{Discussion}

Adult-onset Still's disease, first described in children by George Still in 1896, is an uncommon systemic autoinflammatory disease [4]. Its clinical manifestations are broad-spectrum and vague, making the disease go undiagnosed. Different sets of diagnostic criteria have been approved, with Yamaguchi et al. being most sensitive (96.3\%) and specific (98.2\%) (Table 2) [5].

\begin{tabular}{|c|c|c|}
\hline Major criteria & Minor criteria & Exclusion criteria \\
\hline Fever $>102 \mathrm{~F}$ for $>1$ week & Sore throat & Infection \\
\hline Arthralgia for $>2$ weeks & Lymphadenopathy & Malignancy \\
\hline Iypical rash & Hepatomegaly/splenomegaly & Other rheumatıc diseases \\
\hline \multirow[t]{2}{*}{$\mathrm{WBC}>10,000 / \mathrm{ml}$} & Abnormal liver function tests & \\
\hline & Negative antinuclear antibody and rheumatold factor & \\
\hline
\end{tabular}

TABLE 2: Yamaguchi criteria depicting common diagnostic criteria to help guide the diagnosis WBC: white blood cell

Major criteria include fever $\left(>39^{\circ} \mathrm{C}\right)$, joint pains, transient maculopapular rash mostly visible on the chest during a fever spike, and neutrophilic leukocytosis. Minor criteria consist of sore throat, lymphadenopathy/splenomegaly, deranged liver function tests (aspartate aminotransferase (AST), alkaline phosphatase (ALP), alanine transaminase (ALT)), and negative RA and ANA levels. Diagnosis requires at least five criteria consisting of two major and no exclusion criteria. Classification criteria are useful for research, but it lacks the sensitivity and specificity necessary for clinical diagnosis, although the Yamaguchi criteria have the highest sensitivity for diagnosis.

Pathogenesis is quite uncertain. Both genetic factors and a variety of infectious triggers have been suggested to be important, however, there has been no evidence of an infectious etiology. It is not known whether all patients share the same etiopathogenic factors. The most widely accepted mechanism is auto- 
inflammation, in which a triggering factor(s) stimulate immune response causing the release of proinflammatory cytokines leading to systemic inflammation $[2,6]$. Laboratory tests supporting the diagnosis of AOSD include anemia, leukocytosis, raised lactate dehydrogenase (LDH), ESR, CRP, and ALP [7]. Hyperferritinemia is a characteristic feature of this disease and has been suggested as a serological marker to monitor response to treatment $[4,8]$.

Clinically disease course may be self-limited or monophasic, intermittent or polycyclic systemic, or chronic articular [9]. The former two patterns are more common than the latter [5]. Our patient suffered from monophasic type. The treatment regimen includes corticosteroids as the first line and disease-modifying antirheumatic drugs (DMARDs) reserved as second-line therapy for patients who fail to achieve full remission on corticosteroids, when arthritis is the predominant manifestation, or for those who have evidence of erosive joint disease on hand radiography [10-11]. The patient was started with steroids, which were tapered over a period of one month, and went into remission.

\section{Conclusions}

Adult-onset Still's disease is an uncommon disorder, frequently misdiagnosed due to its overlapping signs and symptoms. It should always be considered in the differential diagnosis for pyrexia of unknown origin. Along with a basic workup of such patients, to rule out common causes of fever, serum ferritin levels should always be sent, which is an important diagnostic marker of adult-onset Still's disease. Serum ferritin concentrations of more than $3,000 \mathrm{ng} / \mathrm{ml}$ and sometimes values greater than $10,000 \mathrm{ng} / \mathrm{ml}$ have been observed. This will not only avoid unnecessary workup but will also help initiate prompt treatment in the form of steroids or biological inhibitors.

\section{Additional Information \\ Disclosures}

Human subjects: Consent was obtained or waived by all participants in this study. Conflicts of interest: In compliance with the ICMJE uniform disclosure form, all authors declare the following: Payment/services info: All authors have declared that no financial support was received from any organization for the submitted work. Financial relationships: All authors have declared that they have no financial relationships at present or within the previous three years with any organizations that might have an interest in the submitted work. Other relationships: All authors have declared that there are no other relationships or activities that could appear to have influenced the submitted work.

\section{References}

1. Giacomelli R, Ruscitti P, Shoenfeld Y: A comprehensive review on adult onset Still's disease . J Autoimmun. 2018, 93:24-36. 10.1016/j.jaut.2018.07.018

2. Feist E, Mitrovic S, Fautrel B: Mechanisms, biomarkers and targets for adult-onset Still's disease . Nat Rev Rheumatol. 2018, 14:603-18. 10.1038/s41584-018-0081-X

3. Gopalarathinam R, Orlowsky E, Kesavalu R, Yelaminchili S: Adult onset Still's disease: a review on diagnostic workup and treatment options. Case Rep Rheumatol. 2016, 2016:6502373. 10.1155/2016/6502373

4. Seung OP, Sulaiman W: Adult-onset Still's disease: a case report. Oman Med J. 2011, 26:e022. 10.5001/omj.2011.96

5. Kadavath S, Efthimiou P: Adult-onset Still's disease-pathogenesis, clinical manifestations, and new treatment options. Ann Med. 2015, 47:6-14. 10.3109/07853890.2014.971052

6. Wang MY, Jia JC, Yang CD, Hu QY: Pathogenesis, disease course, and prognosis of adult-onset Still's disease: an update and review. Chin Med J (Engl). 2019, 132:2856-64. 10.1097/CM9.0000000000000538

7. Agha-Abbaslou M, Bensaci AM, Dike O, Poznansky MC, Hyat A: Adult-onset Still's disease: still a serious health problem (a case report and literature review). Am J Case Rep. 2017, 18:119-24. 10.12659/ajcr.901846

8. Mollaeian A, Chen J, Chan NN, Nizialek GA, Haas CJ: Adult onset Still's disease in the elderly: a case-based literature review. BMC Rheumatol. 2021, 5:12. 10.1186/s41927-021-00183-6

9. Kashfi S, Sharma S, Bengualid V, Sharma S, Gandrabur L: Adult-onset Still's disease in a 28-year-old man from Ghana. Cureus. 2021, 13:e18126. 10.7759/cureus.18126

10. Cavalli G, Farina N, Campochiaro C, Baldissera E, Dagna L: Current treatment options and safety considerations when treating adult-onset Still's disease. Expert Opin Drug Saf. 2020, 19:1549-58. 10.1080/14740338.2020.1839411

11. Sapkota A, Pokhrel N, Adhikari J, Shrestha B, Yadav YK: Adult-onset Still's disease: a case report . JNMA J Nepal Med Assoc. 2020, 58:115-8. 